\title{
Risk of significant traumatic brain injury in adults with minor head injury taking direct oral anticoagulants: a cohort study and updated meta- analysis
}

\author{
Gordon Fuller 이, ${ }^{1}$ Lisa Sabir 이, ${ }^{1}$ Rachel Evans, ${ }^{1}$ Daniel Bradbury, ${ }^{2}$ \\ Maxine Kuczawski 다, 'Suzanne M Mason
}

\begin{abstract}
Handling editor Simon Horne
${ }^{1}$ Center for Urgent and Emergency Care Research, School of Health and Related Research, The University of Sheffield, Sheffield, UK 2Emergency Department, Northern General Hospital, Sheffield Teaching Hospitals, Sheffield, UK
\end{abstract}

\section{Correspondence to}

Dr Gordon Fuller, Center for Urgent and Emergency Care Research, School of Health and Related Research, The University of Sheffield, Sheffield S10 2TG, UK; g.fuller@sheffield.ac.uk

Received 20 November 2019 Revised 23 July 2020 Accepted 13 August 2020 Published Online First 8 September 2020

Check for updates

(c) Author(s) (or their employer(s)) 2020. No commercial re-use. See rights and permissions. Published by BMJ.

To cite: Fuller $\mathrm{G}$, Sabir $\mathrm{L}$, Evans $\mathrm{R}$, et al. Emerg Med J 2020:37:666-673.

\begin{abstract}
Background Patients taking direct oral anticoagulants (DOACs) commonly undergo CT head imaging after minor head injury, regardless of symptoms or signs. However, the risk of intracranial haemorrhage $(\mathrm{ICH})$ in such patients is unclear, and further research has been recommended by the UK National Institute for Health and Care Excellence head injury guideline group.
\end{abstract}

Methods An observational cohort study was performed in the UK South Yorkshire major trauma centre between 26 June and 3 September 2018. Adult patients taking DOACs with minor head injury were prospectively identified, with case ascertainment supplemented by screening of radiology and ED information technology systems. Clinical and outcome data were subsequently collated from patient records. The primary endpoint was adverse outcome within 30 days, comprising: neurosurgery, ICH or death due to head injury. A previously published meta-analysis was updated with the current results and the findings of other recent studies.

Results 148 patients with minor head injury were included (GCS 15, $n=107,72 \%$; GCS 14, $n=41,28 \%$ ). Patients were elderly (median 82 years) and most frequently injured from ground level falls ( $n=142$, $96 \%$ ). Overall risk of adverse outcome was 3.4\% (5/148, 95\% Cl 1.4\% to $8.0 \%)$. Five patients had ICH, of whom one died within 30 days. One patient was treated with prothrombin complex concentrate but no patient received critical care management or underwent neurosurgical intervention. Updated random effects meta-analysis, including the current results and two further recent studies, showed a weighted overall risk of adverse outcome of $3.2 \%$ ( $n=29 / 787,95 \% \mathrm{Cl} 2.0 \%$ to $4.4 \%)$.

Conclusions The risk of adverse outcome following mild head injury in patients taking DOACs appears low. These findings would support shared patient-clinician decision making, rather than routine imaging, following minor head injury while taking DOACs.

\section{INTRODUCTION}

Head injury is responsible for 1.4 million ED attendances annually in the UK. ${ }^{1}{ }^{2}$ Mild head injury, classified as a presenting GCS score of $14-15,34$ is usually self-limiting, with less than $1 \%$ of patients having life-threatening sequelae, or intracranial injuries identified by CT head imaging. ${ }^{15}$ Risk

\section{Key messages}

What is already known on this subject

- Patients taking direct oral anticoagulants (DOACs) commonly undergo CT head imaging after minor head injury regardless of symptoms or signs.

- The risk of significant traumatic brain injury in such patients is unclear.

What this study adds

- In this single centre cohort study of 148 patients taking DOACs the risk of intracranial haemorrhage following mild head injury in patients taking DOACs is $3.4 \%(95 \% \mathrm{Cl} 1.4 \%$ to $8.0 \%)$ and $0 \%(95 \% \mathrm{Cl} 0.0 \%$ to $4.0 \%)$. In patients with a normal GCS, no symptoms and a non-dangerous mechanism of injury.

- An updated random effects meta-analysis of a recent systematic, including the current results and two further recent studies, showed a weighted overall risk of adverse outcome of $3.2 \%$ for patients presenting with GCS 14 or 15 ( $n=787,95 \% \mathrm{Cl} 2.0 \%$ to $4.4 \%$ ).

stratification using clinical decision rules, followed by early CT head scanning to detect intracranial pathology, is the current standard of care for these patients. ${ }^{6}$

Up to $2.4 \%$ of the adult population are receiving anticoagulation therapy, with a concomitant increased risk of sustaining intracranial bleeding after head injury. ${ }^{7}$ Traditionally, warfarin has been the most widely prescribed anticoagulant. ${ }^{7}$ However, more recently, direct oral anticoagulants (DOACs) have been introduced and now represent the most commonly used anticoagulant medications. ${ }^{9} 10$ The risk of adverse outcome following mild head injury when taking a DOAC is uncertain. A recent systematic review reported a 4\% 30-day cumulative incidence of death, neurosurgery or intracranial haemorrhage (ICH). However, the quality of the body of evidence was noted to be low secondary to imprecision, indirectness and high risk of bias and further research was recommended. ${ }^{11}$

Current guidance from the UK National Institute for Health and Care Excellence (NICE) recommends 
routine CT imaging of all patients with mild head injury taking anticoagulants within 8 hours of injury. ${ }^{12}$ This approach may result in unnecessary CT imaging incurring financial costs, prolonged patient stays and increased ED crowding. ${ }^{13}$ This study therefore aimed to estimate the risk of adverse outcome after mild head injury in patients taking DOACs to guide ED management. Specific objectives were to describe patient characteristics and clinical management; to estimate the proportion of patients who suffer an adverse outcome; and explore any subgroups at lower risk of adverse outcome.

\section{METHODS}

\section{Study design and setting}

A single centre observational cohort study was performed in the UK South Yorkshire major trauma centre (Northern General Hospital, Sheffield) between 26 June and 3 September 2018. Results of this cohort study were then used to update a recently published systematic review and meta-analysis. ${ }^{11}$ Study methodology and reporting followed best practice recommendations. ${ }^{14-17}$

\section{Sample}

Consecutive adult patients aged $>16$ years presenting to the ED with mild blunt head injury while taking a DOAC were eligible. Mild head injury was defined as a GCS score of 14-15 consistent with the World Federation of Neurosurgical Societies classification of traumatic brain injury. ${ }^{4}$ Any DOAC currently licenced in the UK was included, comprising direct factor IIa inhibitors (dabigatran) or direct factor Xa inhibitors (rivaroxaban, apixaban, edoxaban). Children, patients with penetrating Traumatic Brain Injury (TBI), or those with a non-traumatic spontaneous ICH were excluded.

Patients were identified using a detailed multi-modal case finding process. Where possible patients were prospectively identified by attending clinicians. Cases ascertainment was supplemented by screening of hospital information technology systems. All CT head imaging requests were hand searched for details of patients meeting inclusion criteria. Electronic coding of ED records were also examined for any cases classified as TBI, head or facial trauma. Case notes were then examined in detail to determine eligibility.

\section{Data collection and outcomes}

Hospital and summary care records were examined by two study investigators with a standardised dataset of demographic, clinical data and outcome data extracted into an electronic database. The primary endpoint of adverse outcome at 30 days was clinically significant TBI defined as a composite measure of neurosurgery, CT detected ICH, readmission to hospital or death due to head injury. This was assessed from hospital records and community general practitioner summary care records for all patients. The routine radiology CT head report was used to assess the presence of ICH.

\section{Analysis}

The analysis proceeded in two stages. First, the cohort study was examined. The characteristics, injuries and clinical management of the cohort were described using summary statistics. The risk of adverse outcome was then calculated with its 95\% CI. The risk of adverse outcome with its $95 \%$ CI was also examined for the subgroup of patients who except for taking an anticoagulant did not otherwise meet NICE clinical or mechanistic imaging criteria (eg, no vomiting, loss of consciousness or neurological deficit $^{6}$; and for those patients who were GCS 15 , asymptomatic and presenting after ground level falls. A sensitivity analysis was performed excluding patients who did not undergo ED CT head imaging.

Second, a recently published systematic review and metaanalysis was updated with results of the current investigation and a contemporary literature search. ${ }^{11}$ The review question was: 'what is the risk of adverse outcome in patients sustaining a mild TBI while anticoagulated with a DOAC?' and a protocol was registered with an international prospective register of systematic reviews (PROSPERO Number CRD42017071411).

Previously identified studies were included that aligned with the current inclusion criteria (ie, adult patients taking DOACs, mild head injury, presenting GCS of 14 or 15). An updated current awareness literature search was also performed to identify any new studies. This used identical search terms and information sources as the original review with search limits from 10 May 2018 to 24 October 2019, as detailed in online supplemental appendix. Article screening, selection, data extraction and risk of bias assessment proceeded as previously described. ${ }^{11}$ Two reviewers screened all citations and independently examined all retrieved full text articles against the inclusion criteria to identify eligible studies. A single reviewer extracted data using a standardised data extraction form; with accuracy checked by a second reviewer. Study authors were contacted where additional information was necessary to assess study eligibility or risk of bias, or obtain relevant results. References were managed in EndNote (Thomson Reuters, California, USA).

A methodological component approach, based on recommendations of the Grades of Recommendation, Assessment, Development and Evaluation Working Group (GRADE) prognosis group ${ }^{18}$ was used to assess risk of bias, comprising the domains of: selection bias, information bias, reporting bias and other sources of bias. Risk of bias in each domain was classified as low, moderate or high, relative to the gold standard of a perfectly performed, unbiased study directly addressing the systematic review question. A single un-blinded reviewer judged the risk of bias in identified studies which were then checked by a second reviewer.

Study specific estimates of risk for eligible studies were compared in Forest plots and heterogeneity assessed subjectively by visual inspection, Cochrane $\mathrm{Q}$ test and the $\mathrm{I}^{2}$ statistic. ${ }^{19}$ A quantitative synthesis was performed, after confirming that studies had relatively homogenous participants and results $\left(\mathrm{I}^{2}<25 \%\right)$. A random effects meta-analysis was subsequently performed using the binomial distribution to model the withinstudy variability and exact binomial 95\% CIs calculated. ${ }^{20}$ The overall quality of evidence for the risk estimate was assessed using the GRADE approach for prognosis research. ${ }^{18}$

All statistical analyses were carried out in Stata V.12.1 (StataCorp), using the metaprop command for the metaanalysis. ${ }^{21}$ The sample size for the study was determined by the fixed recruitment period secondary to available resources. A power analysis was therefore not appropriate and the 95\% CIs around the effect estimates indicate the precision of results.

\section{Public and patient involvement}

The James Lind Alliance identified management of anticoagulated patients sustaining mild head injury as the 12th most important emergency medicine research priority in the UK. The Sheffield Emergency Care forum public and patient involvement group helped develop the research question and approved the study methodology. Results were presented to this group and our 


\begin{tabular}{|c|c|}
\hline Patient characteristic & Summary statistic $n=148$ \\
\hline Age (median, IQR) & 82 years $(79-88)$ \\
\hline Female $(n, \%)$ & $90,60.1 \%$ \\
\hline \multicolumn{2}{|l|}{$\operatorname{DOAC}(\mathrm{n}, \%)$} \\
\hline Apixaban & $64,43.2 \%$ \\
\hline Rivaroxaban & $84,56.8 \%$ \\
\hline \multicolumn{2}{|l|}{ Indication for anticoagulation (n, \%) } \\
\hline AF & $110,74.3 \%$ \\
\hline VTE & $18,12.8 \%$ \\
\hline AF and VTE & $8,5.4 \%$ \\
\hline Other or unclear & $11,7.4 \%$ \\
\hline \multicolumn{2}{|l|}{ Baseline GCS (n, \%) } \\
\hline 15 & $107,72.3 \%$ \\
\hline $14^{*}$ & $41,27.7 \%$ \\
\hline \multicolumn{2}{|l|}{ Performance status } \\
\hline Independent & $60,40.5 \%$ \\
\hline Requires assistance with $\mathrm{ADL}$ & $54,36.5 \%$ \\
\hline Fully dependent ADL & $34,23.0 \%$ \\
\hline \multicolumn{2}{|l|}{ Mobility } \\
\hline Fully independent & $61,41.2 \%$ \\
\hline Independent, requires mobility aid & $73,49.3 \%$ \\
\hline Requires assistance and mobility aid & $14,9.5 \%$ \\
\hline \multicolumn{2}{|l|}{ Comorbidities } \\
\hline None & $0,0 \%$ \\
\hline Single comorbidity & $5,3.4 \%$ \\
\hline Two comorbidities & $18,12.2 \%$ \\
\hline$>3$ comorbidities & $125,84.5 \%$ \\
\hline Dementia & $25,16.9 \%$ \\
\hline DNACPR $(n, \%)$ & $38,25.7 \%$ \\
\hline
\end{tabular}

* Of the 41 patients with a post-injury GCS of 14,16 had a reduced baseline GCS equal to 14 secondary to dementia

$A D L$, Activities of daily living; AF, Atrial fibrillation; DNACPR, Do not attempt cardiopulmonary resuscitation; DOAC, direct oral anticoagulant; VTE, Venous thromboembolism.

dissemination plan involving journal submission and conference presentation agreed.

\section{RESULTS}

\section{Cohort study}

\section{Demographic characteristics}

Over the study period 148 patients with mild head injury taking DOACs presented to the South Yorkshire Major Trauma Centre ED. All patients were followed up successfully for 30 days post injury. The cohort characteristics are detailed in table 1. Patients were elderly (median age 82 years, IQR 79-88), predominately women (59.4\%), had multiple comorbidities (median two chronic medical conditions, IQR 3-4) and poor performance status (59.5\% requiring assistance with activities of living, $62.8 \%$ not independently mobile and $25.7 \%$ with a pre-existing do not resuscitate order). Rivaroxaban was the most commonly taken DOAC (rivaroxaban 56.8\%, apixaban 43.2\%). Atrial fibrillation was the predominant indication for anticoagulation $(74.3 \%)$, followed by venous thromboembolism (12.8\%). Concomitant antiplatelet therapy was rare, occurring in only two patients (1.4\%, one patient taking aspirin and one taking clopidogrel).

\section{Presentation}

The most common mechanism of injury was ground level falls, occurring in $96 \%$ of patients. The majority of patients presented with a GCS of $15(107 / 148,72.3 \%)$. Of the 41 patients with a post-injury GCS of 14 (27.7\%), 16 had a reduced baseline GCS equal to 14 secondary to dementia. The majority of patients were asymptomatic following their head injury (111/148, 75.0\%), with headache being the most commonly endorsed symptom $(15 / 148,10.1 \%)$. External signs of head trauma were absent in $43.9 \%$ of patients $(65 / 148)$, with contusions $(25.0 \%, 37 / 148)$ and lacerations $(21.0 \%, 31 / 148)$ being the most common superficial injuries. Figure 1 and table 2 summarise the cohort's clinical presentations.

\section{Injury, management and outcomes}

Fourteen patients $(9.5 \%)$ did not undergo head imaging, of whom 12 were asymptomatic with no reduction in their normal GCS. Two further patients with dementia and baseline GCS of 14 were unable to tolerate a CT scan. All of these patients were discharged from the ED survived to 30 days and did not re-present to hospital.

The remaining patients underwent CT head imaging $(90.5 \%$, $134 / 148)$. TBI was detected in $3.4 \%$ of patients $(5 / 148)$, comprising one patient with a subdural haematoma, two patients with traumatic subarachnoid haemorrhage, one patient with intracerebral haemorrhage and one patient with concomitant subdural, intracerebral, subarachnoid haemorrhage and occipital skull fracture. All patients with ICH were admitted to hospital as a result of their TBI (median length of stay 4 days, IQR 3-8); three patients under the care of the emergency medicine head injury team and two to general medical wards. No other patients died due to TBI, were re-admitted, or underwent neurosurgical intervention giving a final overall risk of adverse outcome of $3.4 \%(5 / 148,95 \%$ CI 1.4 to 7.9$)$. Excluding patients who did not undergo ED CT head imaging did not substantively alter these results with an overall risk of adverse outcome of $3.7 \%$ (5/134, 95\% CI 1.5 to 8.7$)$.

Anticoagulation was withheld in all patients with TBI and prothrombin complex concentrate administered to one patient. No patient underwent neurosurgery or was admitted to critical care. One patient with ICH died within 30 days as a result of TBI. Patient management is summarised in table 3 .

With the exception of taking an anticoagulant, the majority of patients presenting after head injury did not meet other clinical or mechanistic NICE imaging criteria $(75.7 \%, 112 / 148)$. Of these patients three had ICH $(3 / 112,2.7 \%, 95 \%$ CI $0.0 \%$ to $8.1 \%)$ of whom one had a headache and two had potentially dangerous mechanisms of injury (fall $>2 \mathrm{~m}$, assaulted). No asymptomatic patient with a normal GCS and ground level fall had an adverse outcome $(0 / 91,0 \%, 95 \%$ CI $0.0 \%$ to $4.0 \%)$.

\section{Updated systematic review and meta-analysis}

Four studies from the recently published systematic review and meta-analysis had inclusion criteria (presenting GCS 14-15) aligned with the current investigation. ${ }^{22-25}$ An additional 145 citations were screened for eligibility in the updated literature search, with the full text of 15 articles retrieved for detailed evaluation. During full text examination three eligible observational studies were identified. Selection of studies is summarised in figure 2 .

Spinola et al and Galliazzo et al enrolled study populations aligning with the current study and were included. ${ }^{26}$ These studies were judged to be at an unclear and high risk of bias, respectively. Turcato et al investigated adverse outcome following mild injury in patients taking DOACs, reporting results for patients with GCS $13-15 .^{28}$ Data on outcomes for the patient 


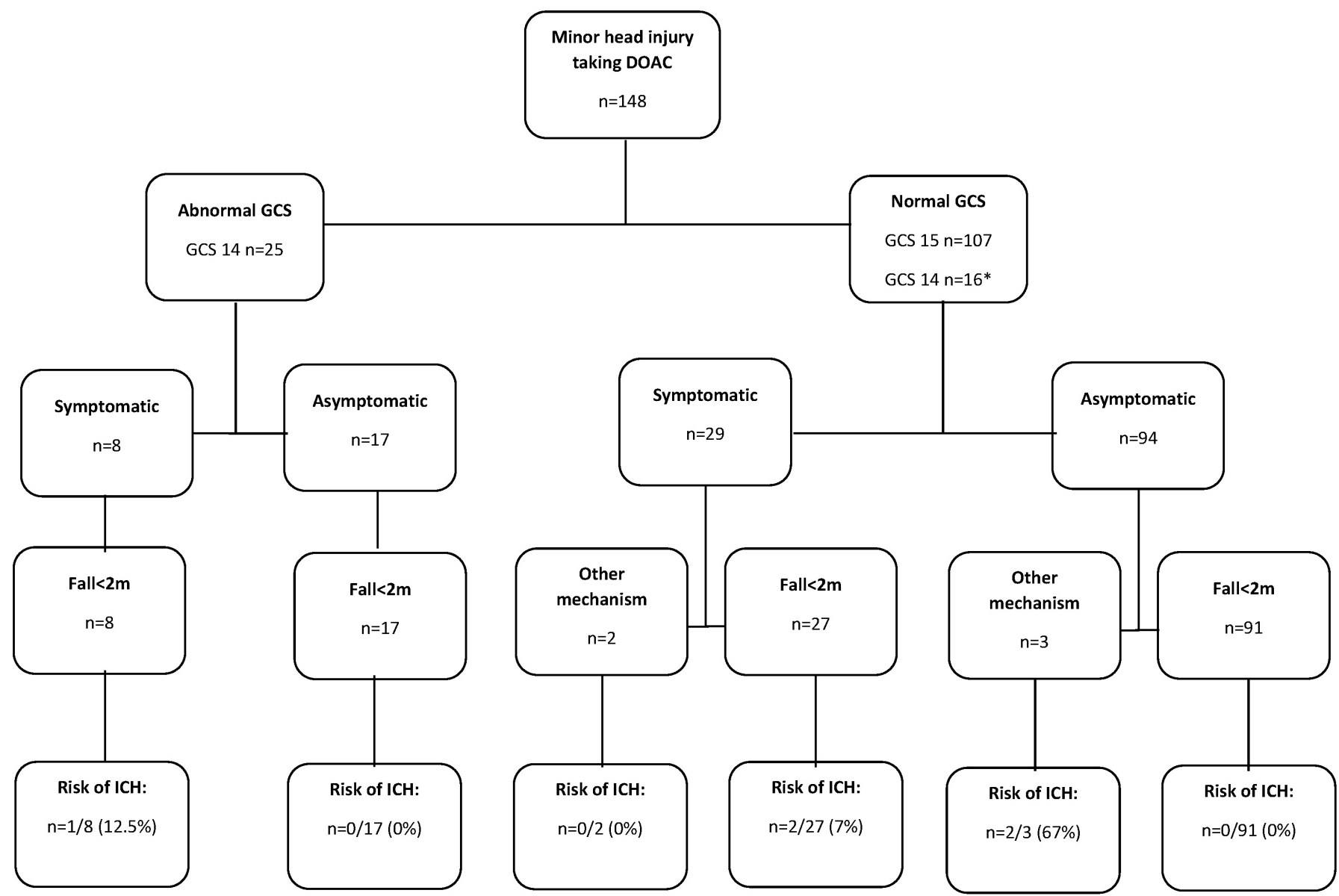

Figure 1 Outcomes of patients taking DOACs following minor head injury stratified by GCS, symptoms and mechanism of injury. DOAC, direct oral anticoagulant; ICH, intracranial haemorrhage.

subgroup with GCS 14 or 15 was requested from the authors allowing inclusion. Risk of bias was judged as high for this study. The characteristics of included studies are summarised in online supplemental appendix.

Estimates of adverse outcome ranged from $0.0 \%$ to $6.4 \%$ across the included studies, as presented in a Forest plot in figure 3. Point estimates for adverse outcome risk varied slightly, with 95\% CIs for each study closely overlapped indicating very homogenous results. The $\mathrm{I}^{2}$ statistic was $0.0 \%$ with a nonsignificant $\mathrm{Q}$ statistic $(\mathrm{p}=0.73)$. A random effects meta-analysis showed a weighted adverse outcome risk of 3.2\% $(n=29 / 787$, $95 \%$ CI $2.0 \%$ to $4.4 \%$ ). The GRADE quality of evidence was downgraded to moderate quality based on: methodology (high or unclear risk of bias) and precision (relatively wide 95\% CI for pooled adverse event estimate). The quality rating was not affected by indirectness, heterogeneity or publication bias.

\section{DISCUSSION}

\section{Summary}

Patients sustaining mild head injury while taking DOACs were elderly, with multiple comorbidities, reduced mobility and poor performance status. Patients commonly underwent CT head imaging after head injury, even in the absence of external signs of head injury, symptoms or abnormal clinical signs. Overall risk of adverse outcome in the current cohort was 3.4\% (5/148, $95 \%$ CI $1.4 \%$ to $8.0 \%$ ), with a pooled risk of adverse outcome of $3.2 \%\left(\mathrm{n}=787,95 \%\right.$ CI $2.0 \%$ to $\left.4.4 \%, \mathrm{I}^{2}=0.0 \%\right)$ in an updated meta-analysis. Five patients had ICH, of whom one died within
30 days. One patient was treated with prothrombin complex concentrate but critical care management or neurosurgical intervention was not performed. Subgroup analyses demonstrated a low risk of adverse outcome in patients not meeting NICE imaging criteria except for taking an anticoagulant $(2.7 \%$, $95 \%$ CI $0.0 \%$ to $8.1 \%$ ), or in asymptomatic, GCS 15 patients with a low energy mechanism of injury $(0 \%, 95 \%$ CI $0.0 \%$ to $4.0 \%)$.

\section{Interpretation of findings}

The benefits of CT imaging in patients following mild head injury include identification of ICH amenable to neurosurgical intervention and risk stratification to inform hospital admission for supportive management and observation. ${ }^{1}$ In the current cohort no patients underwent neurosurgical intervention or critical care admission, likely reflecting the advanced age and poor health status of the study population. However, all patients with ICH did undergo a change in management with cessation of anticoagulation and the administration of prothrombin concentrate complex in one case. This might support the current approach of routine investigation regardless of symptoms or signs.

An alternative strategy could be to forego imaging and withhold anticoagulation temporarily, or permanently. The advanced age, poor performance status and apparent falls risk evident in this cohort may suggest an unfavourable risk/benefit ratio for ongoing DOAC therapy. If anticoagulation was to be stopped in any case, CT head imaging may then be futile and highly unlikely to change management. A large proportion of patients 


\begin{tabular}{|c|c|}
\hline Patient characteristic & Summary statistic $n=148$ \\
\hline \multicolumn{2}{|l|}{ Mechanism of injury } \\
\hline Ground level fall & $142,96.0 \%$ \\
\hline Fall $>2 \mathrm{~m}$ & $3,2.0 \%$ \\
\hline Assault & $2,1.4 \%$ \\
\hline Unknown & $1,0.6 \%$ \\
\hline \multicolumn{2}{|l|}{ Presenting GCS $(n, \%)$} \\
\hline 15 & $107,72.3 \%$ \\
\hline $14^{*}$ & $41,27.7 \%$ \\
\hline \multicolumn{2}{|l|}{ Symptoms } \\
\hline Asymptomatic & $111,75.0 \%$ \\
\hline Headachet & $13,8.8 \%$ \\
\hline Amnesiat & $13,8.8 \%$ \\
\hline LOCt & $6,4.1 \%$ \\
\hline Vomiting $\dagger$ & $2,1.4 \%$ \\
\hline \multicolumn{2}{|l|}{ External signs of head injury } \\
\hline None & $65,43.9 \%$ \\
\hline Abrasion‡ & $12,8.1 \%$ \\
\hline Laceration‡ & $31,20.9 \%$ \\
\hline Contusion/haematomał & $37,25.0 \%$ \\
\hline Boney tenderness $\ddagger$ & $2,1.4 \%$ \\
\hline Epistaxis $¥$ & $2,1.4 \%$ \\
\hline
\end{tabular}

${ }^{*} \mathrm{n}=16$ with a baseline GCS of 14 .

tPatients could have more than one symptom.

¥Patients could have more than one external sign of head injury. LOC, Loss of conciousness.

were admitted to hospital for a non-TBI reason following their head injury (44.1\%, usually for social reasons) despite normal head imaging, which would improve the safety of this approach further with an in-built period of observation.

\begin{tabular}{ll}
\hline \multicolumn{1}{l}{ Table 3 Clinical management and outcomes } \\
\hline Overall patient outcomes & $\mathrm{N}=148$ \\
\hline Outcomes & \\
\hline CT head imaging & $134,90.5 \%$ \\
\hline Yes & $14,9.5 \%$ \\
\hline No & $5,3.4 \%$ \\
\hline Adverse outcome & $5.3 .4 \%$ \\
\hline Overall & - \\
\hline ICH & - \\
\hline Neurosurgery & $1,0.7 \%$ \\
\hline Re-admission & $80,54.1 \%$ \\
\hline Death due to head injury within 30 days & $68,45.9 \%$ \\
\hline Disposal & $\mathrm{n}=5$ \\
\hline Discharged & $5,100.0 \%$ \\
\hline Admittedt & $1,25.0 \%$ \\
\hline Head injury management for patients with ICH & - \\
\hline Anticoagulation stopped & - \\
\hline Beriplex & \\
\hline Neurosurgery & \\
\hline Critical care admission & \\
\hline *Palents cold have more than & \\
\hline
\end{tabular}

*Patients could have more than one endpoint comprising the composite outcome. tPatients admitted for non-head injury reason in $n=63 / 68$ patients. $\mathrm{ICH}$, intracranial haemorrhage.
Three quarters of patients investigated for head injury while taking a DOAC did not meet existing NICE imaging criteria except for taking an anticoagulant, offering the opportunity for development of a dedicated clinical decision rule to tailor imaging decisions in this subgroup. ${ }^{6}$ The risk among these patients was slightly (but not statistically significantly) lower at $2.7 \%$. In each case there was a clinical feature that could suggest higher risk such as a dangerous mechanism of injury or symptoms. The finding of no adverse outcomes in asymptomatic, neurologically intact patients following a ground level fall could support a selective imaging approach, but the upper limit of the adverse outcome risk 95\% CI was 5\% limiting the conclusions that can be drawn in this subgroup.

Although the risk of adverse outcome was low, it is unknown if this is below the maximal acceptable risk threshold of patients and clinicians, notwithstanding the low probability of neurosurgical intervention. Although not well studied, emergency medicine clinicians appear to have low risk tolerance, and have been shown to be willing to accept only a $<1 \%$ of missed acute coronary syndrome, for example. ${ }^{29}$ CT head imaging may also be perceived as important for patient prognostication and to mitigate medico-legal or regulatory risk. However, patients appear willing to accept higher levels of adverse outcome risk, for example up to a $19 \%$ pretest probability of TBI in parents deciding whether a child should undergo head CT after minor head injury. ${ }^{30} 31$ It seems likely that elderly patients might be willing to accept a small risk of an incidental CT head finding in preference to a prolonged ED stay, and a shared decision making model with patients and family may be helpful.

\section{Generalisability}

The recruitment of consecutive cases in an English ED following national head injury guidelines should provide good generalisability to UK patients. However, there may be regional differences in patient demographics or variations in DOAC prescribing patterns that could limit external validity. No patients were included taking dabigatran or edoxaban, which may be prescribed more frequently in other settings, for example, North America. ${ }^{32}$ Bleeding risk with these drugs may be slightly lower than rivaroxaban or apixaban. ${ }^{33}$ The rate of neurosurgical intervention might be higher in younger, healthier, populations. Patient characteristics, practice patterns and medicolegal considerations also differ in other countries challenging international extrapolation of results.

\section{Comparison to existing literature}

A recent systematic review by Fuller et al examined the risk of adverse outcome in patients with mild head injury taking DOACs, although this examined patients with presenting GCS of $13-15 .{ }^{11}$ Included studies were at high or unclear risk of bias and the overall quality of available evidence was consequently low, indicating little confidence in the reported pooled risk estimate. We have updated the literature searches and meta-analysis in the current study for the more relevant population presenting with GCS 14-15. A larger literature is available examining the effects of warfarin. The AHEAD study is the most recent and comprehensive investigation, including 3416 adults who had suffered mild blunt TBI and were currently taking warfarin. ${ }^{13}$ The overall adverse outcome estimate was higher at 5.9\% (95\% CI $5.2 \%$ to $6.7 \%$ ) than the reported pooled result for DOACs. For patients with GCS 15 and no associated symptoms, the risk of adverse outcome was consistent with the current study at $2.7 \%$ (95\% CI 2.1 to 3.6). Given the paucity of available data it 


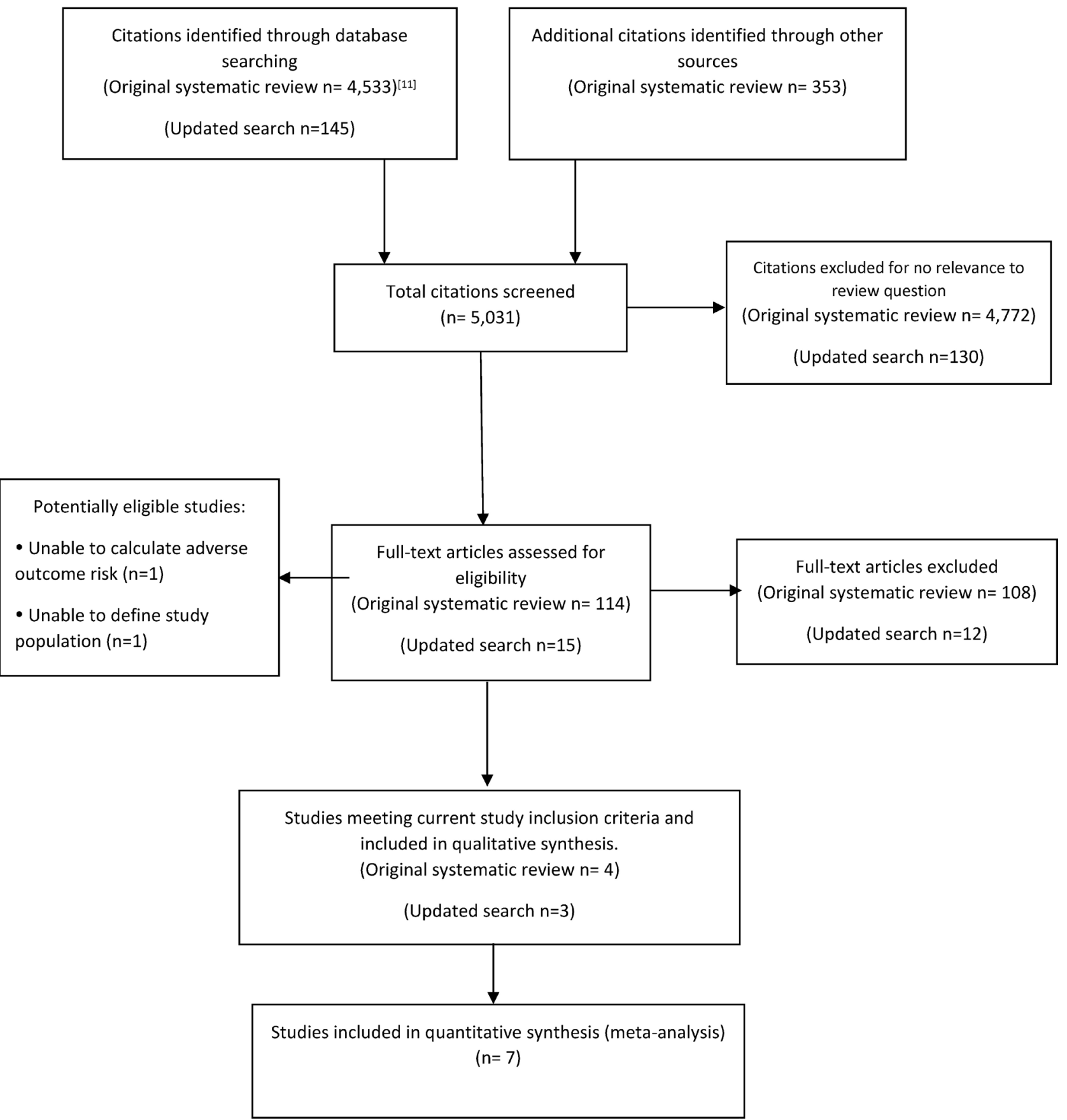

Figure 2 Identification and selection of studies for inclusion in the systematic review.

is not possible to say conclusively whether the adverse outcome risk differs between warfarin and DOACs.

Antiplatelet therapy is common in patients sustaining mild head injury, but the risk of significant TBI following mild head injury is unclear. A contemporary systematic review by van den van den Brand et al suggested increased odds $(2.72$, 95\% CI 1.92 to 3.85 ) of traumatic ICH associated with antiplatelet use, although there was significant heterogeneity and risk of bias in the included studies. ${ }^{34}$ More recently, a large US prospective study reported no increase in risk from aspirin or clopidogrel monotherapy following mild head injury. ${ }^{35}$ Conversely, dual aspirin/clopidogrel appeared to confer a significant risk of $\mathrm{ICH}$ or neurosurgery $(10.6 \%, 95 \%$ CI 5.7 to 18.9$)$, which was higher than that observed for DOACs in the current meta-analysis.
Combined treatment with antiplatelet agents and anticoagulant therapy is less common, and there is a paucity of evidence on bleeding risk following mild head injury.

\section{Limitations}

The cohort study has a number of strengths including comprehensive case ascertainment, consecutive recruitment, detailed data collection and a relevant primary outcome of significant TBI. However, there are limitations that could affect the internal validity and interpretation of results. First, available resources restricted the study to a single centre with consequently restricted sample size. Despite being the second largest reported international cohort, and largest UK study, the analysis is therefore 


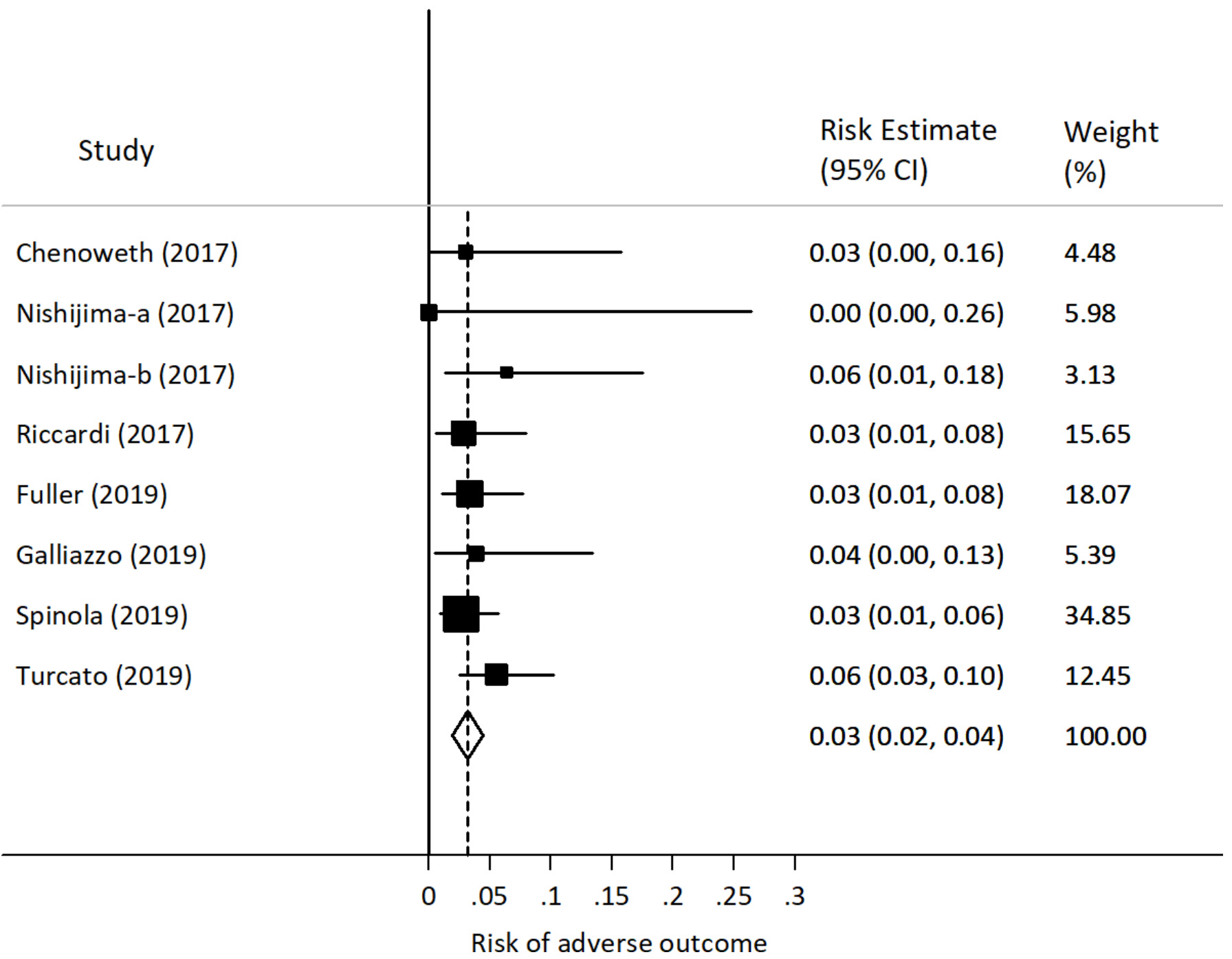

Figure 3 Forest plot presenting individual and pooled risk of adverse outcome following mild head injury while taking direct oral anticoagulants. Dots present point estimates, shaded boxes indicate study weights and whiskers represent $95 \% \mathrm{Cl}$.

relatively underpowered with wide 95\% CI widths; and may have limited generalisability. The low numbers of patients with adverse outcomes also precluded examination of predictors of adverse outcome, risks from different DOACs or investigation of subgroups. However, using the current study findings in an updated meta-analysis provides a precise risk estimate for use in clinical practice. A definitive large-scale multicentre study is required to corroborate these findings.

Second, some data collection was obtained through chart review with its inherent problems. ${ }^{15} 1636$ Third, follow-up was limited to 30 days and it is possible, but unlikely, that delayed deterioration occurred for example, development of a chronic subdural haematoma. Fourth, a small number of patients did not receive ED CT head scanning, and it is possible that $\mathrm{ICH}$ went undetected in these patients. Exclusion of these patients from consideration did not materially change results; and the composite outcome, including death and hospital readmission, suggests that any such cases did not have clinically important TBI. Finally, different DOAC dosing regimens, for example, for venous thromboembolism prophylaxis, were not examined.

The updated systematic review followed Cochrane Collaboration and Preferred Reporting Items for Systematic Reviews and Meta-Analyses guidelines which should ensure that all relevant evidence was included, accurately and precisely coded, validly assessed for risk of bias and impartially analysed and interpreted. However, quantitative synthesis of homogenous studies at high or unclear risk of systematic error could produce precise, but erroneous results secondary to underlying biases

\section{CONCLUSIONS}

The risk of adverse outcome following mild head injury in patients taking DOACs is relatively low, particularly in patients with a normal GCS, no symptoms and a non-dangerous mechanism of injury. Only one patient received a substantive change in management as a result of CT head imaging and no patient with ICH underwent neurosurgery or received critical care. These findings would support shared patient-clinician decision making, rather than routine imaging, following minor head injury while taking DOACs.

Twitter Rachel Evans @masons301265 and Suzanne M Mason @ProfSueMason

Contributors All authors made substantial contributions to the conception and design (GF, RE, LS, DB, MK, SMM), acquisition of the data (RE, LS, DB) or analysis and interpretation (GF, SMM). GF drafted the article and all other authors revised it critically for important intellectual content. SMM is the guarantor. All authors had full access to all of the data in the study and can take responsibility for the integrity of the data and the accuracy of the data analysis. 
Funding The authors have not declared a specific grant for this research from any funding agency in the public, commercial or not-for-profit sectors.

Competing interests None declared.

Patient and public involvement Patients and/or the public were involved in the design, or conduct, or reporting, or dissemination plans of this research. Refer to the Methods section for further details.

Patient consent for publication Not required.

Ethics approval The study received approval from the Sheffield Teaching Hospitals Ethics committee (ethics approval reference STH20253). A pre-specified protocol was developed prior to analysis.

Provenance and peer review Not commissioned; externally peer reviewed.

Data availability statement Data are available upon reasonable request. All data requests should be submitted to the corresponding author for consideration. Access to anonymised data may be granted following review.

\section{ORCID iDs}

Gordon Fuller http://orcid.org/0000-0001-8532-3500

Lisa Sabir http://orcid.org/0000-0001-6488-3314

Maxine Kuczawski http://orcid.org/0000-0002-0774-8113

Suzanne M Mason http://orcid.org/0000-0002-1701-0577

\section{REFERENCES}

1 Pandor A, Goodacre S, Harnan S, et al. Diagnostic management strategies for adults and children with minor head injury: a systematic review and an economic evaluation. Health Technol Assess 2011:15:1-202.

2 Kay A, Teasdale G. Head injury in the United Kingdom. World J Surg 2001;25:1210-20.

3 Vos PE, Alekseenko Y, Battistin L, et al. Mild traumatic brain injury. Eur J Neurol 2012;19:191-8

4 Servadei F, Teasdale G, Merry G, et al. Defining acute mild head injury in adults: a proposal based on prognostic factors, diagnosis, and management. J Neurotrauma 2001;18:657-64.

5 Stein SC, Ross SE. The value of computed tomographic scans in patients with low-risk head injuries. Neurosurgery 1990;26:638-40.

6 Hodgkinson S, Pollit V, Sharpin C, et al. Early management of head injury: summary of updated NICE guidance. BMJ 2014;348:g104.

7 Pirmohamed M. Warfarin: almost 60 years old and still causing problems. Br J Clin Pharmacol 2006;62:509-11.

8 Stewart A, Ganguli A, FitzGerald R, et al. Variation in warfarin prescribing and dosing in the UK: a national survey of anticoagulation clinics. J Clin Pharm Ther 2015;40:466-71.

9 Loo SY, Dell'Aniello S, Huiart L, et al. Trends in the prescription of novel oral anticoagulants in UK primary care. Br J Clin Pharmacol 2017;83:2096-106.

10 Barnes GD, Lucas E, Alexander GC, et al. National trends in ambulatory oral anticoagulant use. Am J Med 2015;128:1300-5.

11 Fuller GW, Evans R, Preston L, et al. Should Adults With Mild Head Injury Who Are Receiving Direct Oral Anticoagulants Undergo Computed Tomography Scanning? A Systematic Review. Ann Emerg Med 2019;73:66-75.

12 National Institute for Health and Care Excellence. Head injury: assessment and early management (NICE guideline). London: National Institute for Health and Care Excellence, 2019

13 Mason S, Kuczawski M, Teare MD, et al. AHEAD study: an observational study of the management of anticoagulated patients who suffer head injury. BMJ Open 2017;7:e014324.

14 von Elm E, Altman DG, Egger M, et al. The strengthening the reporting of observational studies in epidemiology (STROBE) statement: guidelines for reporting observational studies. J Clin Epidemiol 2008;61:344-9.
15 Gearing RE, Mian IA, Barber J, et al. A methodology for conducting retrospective chart review research in child and adolescent psychiatry. J Can Acad Child Adolesc Psychiatry 2006;15:126-34.

16 Kaji AH, Schriger D, Green S. Looking through the retrospectoscope: reducing bias in emergency medicine chart review studies. Ann Emerg Med 2014;64:292-8.

17 Vassar M, Holzmann M. The retrospective chart review: important methodological considerations. J Educ Eval Health Prof 2013;10:12

18 Higgins JPT, Thompson SG. Quantifying heterogeneity in a meta-analysis. Stat Med 2002;21:1539-58

19 Iorio A, Spencer FA, Falavigna M, et al. Use of GRADE for assessment of evidence about prognosis: rating confidence in estimates of event rates in broad categories of patients. BMJ 2015;350:h870.

20 Borenstein M, Hedges LV, Higgins JPT, et al. Introduction to meta-analysis. Chichester, UK: Wiley, 2009.

21 Nyaga VN, Arbyn M, Aerts M. Metaprop: a Stata command to perform meta-analysis of binomial data. Arch Public Health 2014;72:39.

22 Chenoweth JA, Johnson MA, Shook L, et al. Prevalence of intracranial hemorrhage after blunt head trauma in patients on Pre-injury dabigatran. West J Emerg Med 2017:18:794-9.

23 Nishijima DK, Gaona SD, Waechter T, et al. Out-of-Hospital Triage of Older Adults With Head Injury: A Retrospective Study of the Effect of Adding "Anticoagulation or Antiplatelet Medication Use" as a Criterion. Ann Emerg Med 2017;70:127-38.

24 Nishijima DK, Gaona SD, Waechter T, et al. The incidence of traumatic intracranial hemorrhage in head-injured older adults transported by EMS with and without anticoagulant or antiplatelet use. J Neurotrauma 2017. doi:10.1089/neu.2017.5232. [Epub ahead of print: 06 Nov 2017].

25 Riccardi A, Spinola B, Minuto P, et al. Intracranial complications after minor head injury (MHI) in patients taking vitamin $\mathrm{K}$ antagonists (VKA) or direct oral anticoagulants (DOACs). Am J Emerg Med 2017;35:1317-9.

26 Spinola MB, Riccardi A, Minuto P, et al. Hemorrhagic risk and intracranial complications in patients with minor head injury (MHI) taking different oral anticoagulants. Am J Emerg Med 2019:37:1677-80.

27 Galliazzo S, Bianchi MD, Virano A, et al. Intracranial bleeding risk after minor traumatic brain injury in patients on antithrombotic drugs. Thromb Res 2019:174:113-20.

28 Turcato G, Zannoni M, Zaboli A, et al. Direct oral anticoagulant treatment and mild traumatic brain injury: risk of early and delayed bleeding and the severity of injuries compared with vitamin K antagonists. J Emerg Med 2019;57:817-24.

29 Than M, Herbert M, Flaws D, et al. What is an acceptable risk of major adverse cardiac event in chest pain patients soon after discharge from the emergency department?: a clinical survey. Int J Cardiol 2013;166:752-4.

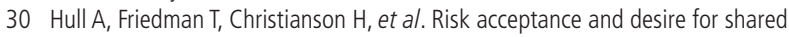
decision making in pediatric computed tomography scans: a survey of 350. Pediatr Emerg Care 2015;31:759-61.

31 Padalecki J, Xu KT, Smith C, et al. How much risk are emergency department patients willing to accept to avoid diagnostic testing. Turk J Emerg Med 2017;17:16-21.

32 O'Neal WT, Sandesara PB, Claxton J'Neka S, et al. Provider specialty, anticoagulation prescription patterns, and stroke risk in atrial fibrillation. J Am Heart Assoc 2018;7:e007943

33 Wolfe Z, Khan SU, Nasir F, et al. A systematic review and Bayesian network metaanalysis of risk of intracranial hemorrhage with direct oral anticoagulants. J Thromb Haemost 2018;16:1296-306.

34 van den Brand CL, Tolido T, Rambach AH, et al. Systematic review and meta-analysis: is Pre-Injury antiplatelet therapy associated with traumatic intracranial hemorrhage? J Neurotrauma 2017;34:1-7.

35 Probst MA, Gupta M, Hendey GW, et al. Prevalence of intracranial injury in adult patients with blunt head trauma with and without anticoagulant or antiplatelet use. Ann Emerg Med 2020;75:354-64.

36 Vassar M, Holzmann M. The retrospective chart review: important methodological considerations. J Educ Eval Health Prof 2013;10:12-13. 